\title{
Multi-Infarct Dementia
}

National Institute of Neurological Disorders and Stroke (NINDS)

\section{Source}

National Institute of Neurological Disorders and Stroke (NINDS). Multi-Infarct Dementia

Information Page.

Multi-infarct dementia (MID) is a common cause of memory loss in the elderly. MID is caused by multiple strokes (disruption of blood flow to the brain). Disruption of blood flow leads to damaged brain tissue. Some of these strokes may occur without noticeable clinical symptoms. Doctors refer to these as "silent strokes." An individual having a silent stroke may not even know it is happening, but over time, as more areas of the brain are damaged and more small blood vessels are blocked, the symptoms of MID beg in to appear. MID can be diagnosed by an MRI or CT of the brain, along with a neurological examination. Symptoms include confusion or problems with short-term memory; wandering, or getting lost in familiar places; walking with rapid, shuffling steps; losing bladder or bowel control; laughing or crying inappropriately; having difficulty following instructions; and having problems counting money and making monetary transactions. MID, which typically begins between the ages of 60 and 75, affects men more often than women. Because the symptoms of MID are so similar to Alzheimer's disease, it can be difficult for a doctor to make a firm diagnosis. Since the diseases often occur together, making a single diagnosis of one or the other is even more problematic. 